\title{
COMPARISON OF EFFECTIVENESS OF PATIENT CONTROLLED ANALGESIA AND PAIN PUMP VERSUS PATIENT CONTROLLED ANALGESIA FOLLOWING SURGERY FOR ADOLESCENT IDIOPATHIC SCOLIOSIS
}

\author{
(1) Sinan YILAR¹, (1) Ali AHISKALIOĞLU2 \\ ${ }^{1}$ Atatürk University Faculty of Medicine, Department of Orthopedics and Traumatology, Erzurum, Turkey \\ ${ }^{2}$ Atatürk University Faculty of Medicine, Department of Anesthesiology and Reanimation, Erzurum, Turkey
}

\begin{abstract}
Objective: The pain control is a difficult and tedious process following a surgery for adolescent idiopathic scoliosis (AIS). Although there are many treatment methods used to relieve pain, the pain is not completely controlled yet. This study was aimed to compare the effectiveness of intravenous patient controlled analgesia (PCA) and a combination of PCA and pain pump (PP) (PP + PCA) used after surgery for AIS.

Materials and Methods: In the present study, the results of patients at the age of 12 to 22 years, who had an AIS surgery between 2016 and 2019 at our clinic, were retrospectively reviewed. The patients' postoperative pain scores, need for opioids, time to walk, and discharge time were compared.

Results: The results of 83 patients (Group PCA, $n=34$; Group PP + PCA, $n=49$ ) that met the study criteria were compared. The visual analogue scale (VAS) scores at the postoperative $6^{\text {th }}$ and $12^{\text {th }}$ hours were lower in the group PP $+P C A(p<0.001)$, but there were no differences in pain scores at $24^{\text {th }}$ and $48^{\text {th }}$ hours between the groups ( $p>0.05$ ). The time to walk for the group PP + PCA was significantly earlier than for the group PCA (2.67 \pm 0.99 vs. $3.68 \pm 0.94, p<0.0001)$. As for discharge time, the group $P P+P C A$ was discharged earlier than the group PCA $(8.00 \pm 2.03$ vs. $10.00 \pm 4.56, p=0.045)$. With regard to the postoperative use of opioids, the use by the group PP + PCA was less than the group PCA at the end of both $24^{\text {th }}$ hour and $48^{\text {th }}$ hour ( $p<0.001)$.

Conclusion: Following surgery for AIS, PP + PCA is a good choice for postoperative analgesia in the early postoperative period (the first 12 hours), reducing postoperative use of opioids and allowing patients to walk and to be discharged earlier.

Keywords: Adolescent idiopathic scoliosis, local anesthetic infusion, postoperative analgesia, catheter, pain
\end{abstract}

\section{INTRODUCTION}

Due to recent advances in regional anesthesia options, the pain can be readily controlled following many orthopedic surgeries. However, pain control is still difficult in spinal surgery and the pain cannot be completely controlled yet. The pain control by the use of paracetamol or non-steroidal anti-inflammatory drugs is not sufficient; therefore, there are many pain control systems that have been developed. The methods commonly used for pain control include patient controlled analgesia (PCA), intrathecal opioids, epidural analgesia, wound site infiltration, and catheters inserted into the incision site for continuous release of local anesthetics. The use of intravenous PCA following a scoliosis surgery is a routine procedure that is carried out by many clinics. Many studies showed that the use of PCA with intrathecal or epidural analgesics achieved better pain control as compared to the use of PCA alone ${ }^{(4,9,10)}$. However, as these analgesia options result in postoperative urinary retention ${ }^{(7)}$, delay ambulation and prolong the hospital stay ${ }^{(1)}$, lead to postoperative leakage of cerebrospinal fluid(9,10), and mask the postoperative neurological examination of patients $\mathrm{s}^{(5)}$, they are not often chosen by the surgical teams. Although continuous release of local anesthetics through catheter, which has been widely used in recent years, provides pain control following many surgical procedures ${ }^{(3,13,14)}$, only one study has indicated the use of this method for pain control following an adolescent idiopathic scoliosis (AIS) surgery ${ }^{(11)}$. The objective of this study was to compare the postoperative pain results of patients using PCA alone and patients using PCA + pain pump (PP) following an AIS surgery. Our hypothesis was that the use of intravenous PCA with incisional PP following an AIS surgery would reduce the patient's pain score, use of opioids, and hospital stay due to early mobilization.

Address for Correspondence: Sinan Yılar, Atatürk University Faculty of Medicine, Department of Orthopedics and Traumatology, Erzurum, Turkey E-mail: sinan_yilar@hotmail.com Received: 14.08.2019 Accepted: 23.09.2017

ORCID ID: orcid.org/0000-0002-1268-170X 


\section{MATERIALS AND METHODS}

The approval of faculty's ethics committee was obtained for this study, and 83 patients who underwent a posterior instrumentation and fusion surgery for elective AIS between 2016 and 2019, were at the age of 11 to 22 years, had no known story of cardiac, renal, hepatic or hematologic disorders, peptic ulcer, gastrointestinal hemorrhage, and chronic pain, and did not use analgesics routinely or within the last 24 hours were included in the study. The patients who underwent a procedure other than the planned surgical intervention, were operated by a different surgical team, had a revision surgery, had missing data in the PCA follow-up form and postoperative study data, had no pain scores recorded, had a PCA connected for less than 48 hours, had a drug dependency, were using chronic analgesics, had a central and peripheral neurological disorder, had coagulopathy or were using an anti-coagulant drug, were not cooperative, and had an allergy to any of the drugs used in the study were excluded from the study. To collect the patient data, the PCA follow-up records kept by the department of anesthesiology and reanimation, which were periodically reviewed and stored, and the patient records maintained in our orthopedics clinics during the postoperative period of patients were used.

For all the patients, the postoperative pain score, time to walk, discharge time, and number of opioids used were recorded, and the values of the two groups were compared.

\section{Surgical Technique}

All the patients were operated by the same surgical team; the same incision opening and closure techniques were used; and all the patients were instrumented by pedicle screws. The facet joints of all the patients were removed during the operation and all the patients underwent a fusion with an animal-derived bone graft. During the closure procedure, the end of a drain was inserted into the left side, to proximal, and the other end of the drain was inserted into the right side, to distal. The PP was placed to the right along the incision between the paraspinal muscles (Figure 1).

\section{Anesthesia Technique}

The anesthesia was induced with propofol $\left(2 \mathrm{mg} \mathrm{kg}^{-1}\right)$, fentanyl (1-2 $\left.\mathrm{\mu g} \mathrm{kg}^{-1}\right)$, and rocuronium $\left(0.6 \mathrm{mg} \mathrm{kg}^{-1}\right)$ and maintained by $1-2 \%$ sevoflurane, and the mixture of $50 \% \mathrm{O}_{2}$ and $50 \% \mathrm{~N}_{2} \mathrm{O}$. $2.5 \mathrm{mg}$ of neostigmine and $1 \mathrm{mg}$ of atropine were used after the operation to antagonize the effect of muscle relaxation. The patients were then extubated and transferred to the postanesthetic care unit.

\section{Postoperative Analgesia}

The patients of both groups received $50 \mathrm{mg}$ of dexketoprofen and $1000 \mathrm{mg}$ of paracetamol 30 minutes before the completion of surgery. Dexketoprofen was postoperatively re-administered every 12 hours and paracetamol was postoperatively readministered every 6 hours. A PCA device was connected to the patients after the surgery in the recovery room. The PCA device was prepared with fentanyl and programmed at the concentration of $10 \mu \mathrm{g} / \mathrm{mL}$, with a loading dose of $50 \mu \mathrm{g}$, locked time of 15 minutes, $25 \mu \mathrm{g}$ bolus, and $25 \mu \mathrm{g}$ basal infusion. The PCA was maintained for 48 hours. The patients with VAS score equal to or over 4 were given $25 \mathrm{mg}$ of meperidine and were recorded in the recovery room. The same multimodal analgesia protocol was used for the postoperative analgesia in both groups. The patients with Modified score equal to or over 9 were transferred to the service. The postoperative follow-up and assessment of patients were performed by a researcher

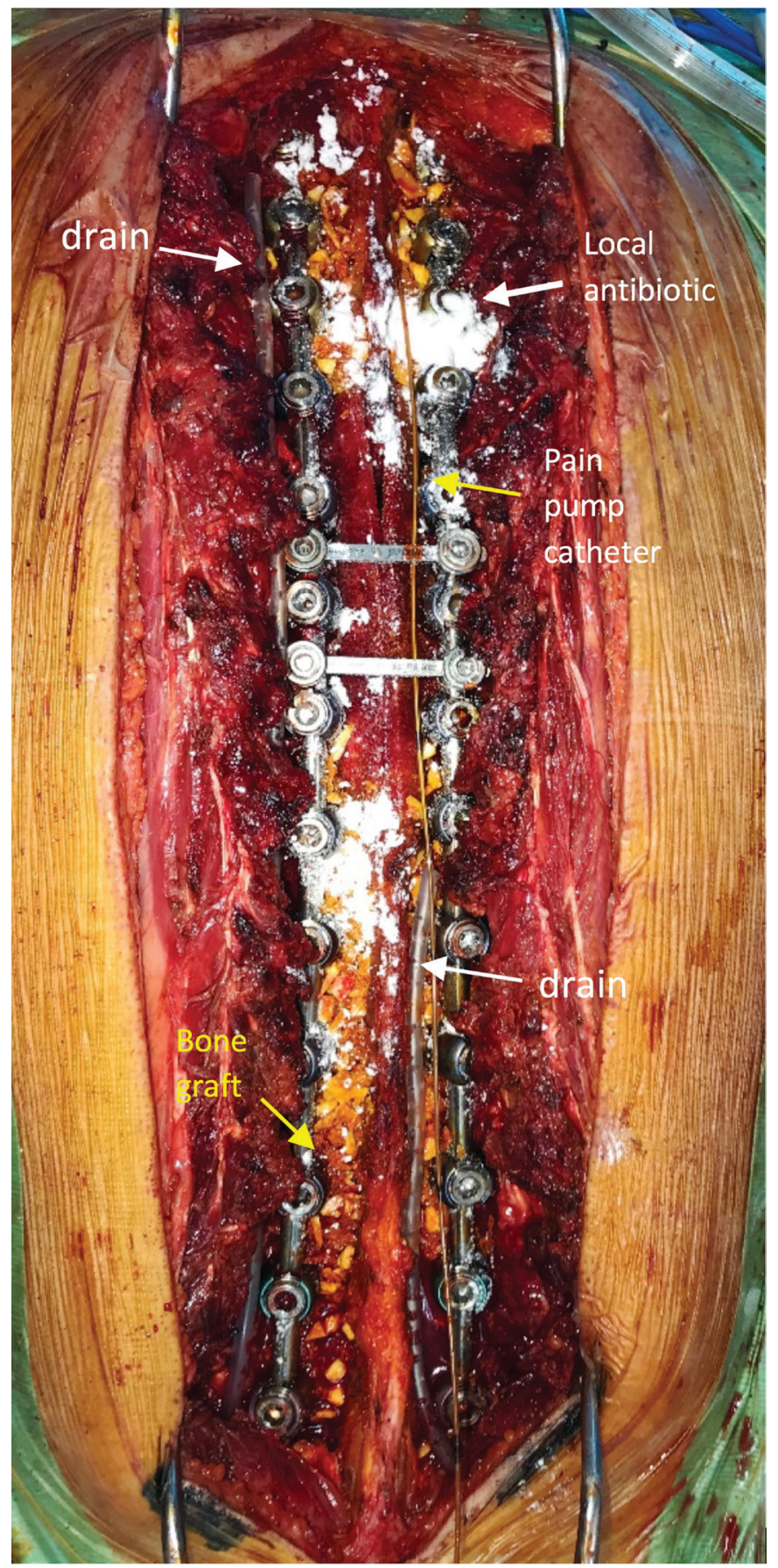

Figure 1. Pain pump and drain placement 
who was unaware of the study group. The postoperative pain was assessed by the visual analogue scale (VAS) score (VAS $0=$ no pain, VAS $10=$ worst possible, unbearable pain). The pain scores were assessed at the postoperative $6^{\text {th }}, 12^{\text {th }}, 24^{\text {th }}$, and $48^{\text {th }}$ hours. The postoperative consumption of opioids was recorded at the end of $24^{\text {th }}$ and $48^{\text {th }}$ hours.

\section{Use of Pain Pump}

ON-Q PainBuster Post-Op Pain Relief System was used as a PP. A balloon contained in the system that retained fluid up to $400 \mathrm{~mL}$ was filled with $0.5 \%$ bupivacaine (Figure 2). $10 \mathrm{~mL}$ of bupivacaine was infused into the incision site through a $1 \mathrm{~mm}$ catheter per hour. The fluid in this pump was consumed at the end of approximately 40 hours. The drain was kept at positive pressure to avoid the increase in the postoperative hemorrhage and suction of anesthetic agents. The PP was removed at the end of 40 hours as it completed the release at the end of 40 hours.

\section{Statistical Analysis}

IBM SPSS 20.0 (SPSS Inc., Chicago, Illinois, USA) was used for statistical assessment. The Histogram and KolmogorovSmirnov tests were used to determine the normal distribution of data. The descriptive data were provided as mean \pm standard deviation. The categorical variables were assessed by the chi-square test. The Student's t-test was used for normally distributed data whereas the Mann-Whitney $U$ test was used for the assessment of data that did not show normal distribution. The value of $p<0.05$ was considered as statistically significant.

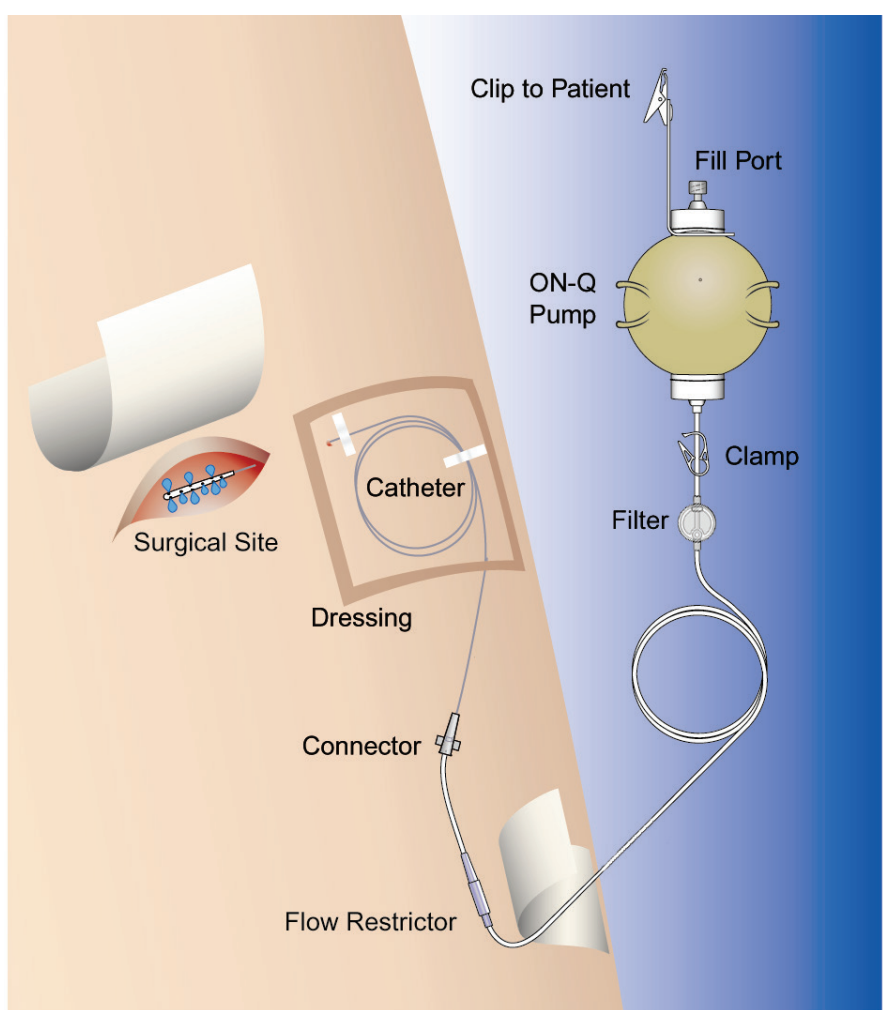

Figure 2. The schematic depiction of the placement of the pain pump

\section{RESULTS}

The results of 122 patients with AIS in the age range of study were reviewed retrospectively. Of 83 patients that met the study criteria, 49 patients received PP + PCA, and 34 patients received PCA alone. There were no differences in preoperative ages, gender, weight, Cobb angle, and fusion level between the two groups (Table 1). The VAS scores at the postoperative $6^{\text {th }}$ hour [7.54 (7-10) vs. $9.53(8-10)]$ and $12^{\text {th }}$ hour [6.21 (5-8) vs. 8.34 (6-9)] were lower in the group PP + PCA than that in the group PCA $(p<0.001)$. There were no differences in the pain

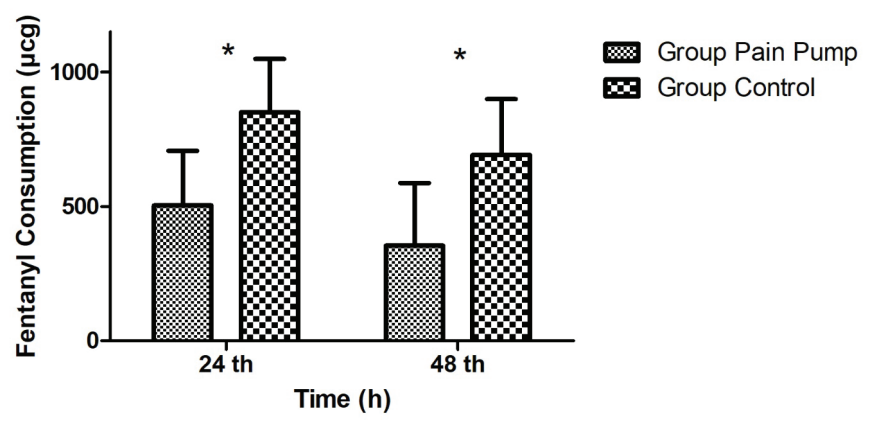

Figure 3 . Opioid consumptionat postoperative $24^{\text {th }}$ and $48^{\text {th }}$ hours

Table 1. Demographic data of patients

\begin{tabular}{|c|c|c|c|}
\hline & $\begin{array}{l}\text { Group } \\
\text { pain pump } \\
(n=49)\end{array}$ & $\begin{array}{l}\text { Group } \\
\text { control } \\
(n=34)\end{array}$ & $\mathbf{p}$ \\
\hline Age (year) & $17.31 \pm 3.20$ & $16.76 \pm 3.03$ & $0.326^{a}$ \\
\hline Gender (M/F) & $16 / 33$ & $7 / 27$ & $0.319^{b}$ \\
\hline Weight (kg) & $59.98 \pm 8.13$ & $58.62 \pm 8.86$ & $0.472^{c}$ \\
\hline ASA (I/II) & $44 / 5$ & $29 / 5$ & $0.733^{b}$ \\
\hline Cobb angle & $64.12 \pm 15.64$ & $64.79 \pm 15.44$ & $0.847^{c}$ \\
\hline Fusion level & $12.82 \pm 1.70$ & $12.76 \pm 2.49$ & $0.285^{a}$ \\
\hline Walking time (day) & $2.67 \pm 0.99$ & $3.68 \pm 0.94$ & $<0.0001^{a}$ \\
\hline Discharge time (day) & $8.00 \pm 2.03$ & $10.00 \pm 4.56$ & $0.045^{a}$ \\
\hline
\end{tabular}

Table 2. Pain scores visual analogue pain scale and wongbaker faces scale

\begin{tabular}{|c|c|c|c|}
\hline & $\begin{array}{l}\text { Group pain pump } \\
(n=49)\end{array}$ & $\begin{array}{l}\text { Group control } \\
(n=34)\end{array}$ & $p^{a}$ \\
\hline VAS $6^{\text {th }}$ hour & $8(7-10)$ & $9.5(8-10)$ & 0.001 \\
\hline VAS $12^{\text {th }}$ hour & $6(5-8)$ & $8(6-9)$ & 0.003 \\
\hline VAS $24^{\text {th }}$ hour & $4(4-6)$ & $4(3-6)$ & 0.692 \\
\hline VAS $48^{\text {th }}$ hour & $3(2-5)$ & $3(2-4)$ & 0.925 \\
\hline
\end{tabular}

Values are presented as median (percentage 25-75) or number, VAS:

Visual analogue scale

a Mann-Whitney U test 
turkishspine

scores at $24^{\text {th }}$ hour $[4.22(4-6)$ vs. $4.45(3-6)]$ and $48^{\text {th }}$ hour [3.12 $(2-5)$ vs. 3.17 (2-4)] between the groups ( $p>0.05$ ) (Table 2).

The time to walk for the group PP + PCA was significantly earlier than the group PCA $(2.67 \pm 0.99$ vs. $3.68 \pm 0.94, p<0.0001)$. As for the discharge time, the group PP + PCA was discharged earlier than the group PCA $(8.00 \pm 2.03$ vs. $10.00 \pm 4.56, p=0.045)$, (Table 3). The number of opioids used was less in the group PP + PCA than in the group PCA at the end of postoperative 24 hours $(503.27 \pm 203.65$ vs. $850.88 \pm 198.44)$ and 48 hours $(354.08 \pm 233.36$ vs. $691.47 \pm 207.92)(p<0.001)$, (Table 4, Figure 3).

Two patients in the group PP + PCA and one patient in the group PCA had a prolonged wound site leakage which was improved without intervention. The superficial wound site infection was treated with oral antibiotics in two patients, each in two groups.

\section{DISCUSSION}

Although epidural analgesia or peripheral nerve blocks help to achieve a good pain control following many orthopedic operations, the pain control following posterior spinal fusion (PSF) is still difficult in the patients with AIS. Although the use of PCA for pain control following PSF is now an indispensable procedure, different methods have been included in the administration of PCA to relieve the patient's pain as its efficacy is insufficient. The PCA is widely used with intrathecal morphine injection (IMI) and epidural catheter infusion (EPI) as an analgesic method. There are many studies that compared these methods with each other or with PCA alone. Some of these studies suggested that IMI + PCA and EPI + PCA were superior to PCA ${ }^{(4,6,6,10)}$. However, as this is an invasive procedure and several complications such as the higher rate of failure to insert a catheter into the epidural space ${ }^{(4,15)}$, causing postoperative leakage of dura mater fluid $^{(4,9,15)}$, leading to respiratory depression ${ }^{(10)}$, and masking the postoperative neurological examination ${ }^{(2,12)}$, may occur, it is not chosen by some clinicians. Another method that can be used in addition to PCA is a PP that is placed in the incision site and capable of continuous release. This method allows continuous release of local anesthetics into the incision site without invasive intervention and it is unlikely to cause side effects that may occur with epidural anesthesia. The most important disadvantages include that a second foreign object is inserted into the wound site in addition to the drain in the postoperative period and a proper fixing cannot be achieved because the catheter is removed from the incision site; therefore it can easily come away during walking and transfer. In the literature, there are limited studies that used a PP following a scoliosis surgery although it was used for different surgeries ${ }^{(11)}$. In a study assessing 244 patients following AIS surgery, there were no differences in pain scores of patients that used and did not use PP at the $6^{\text {th }}, 12^{\text {th }}$ and $24^{\text {th }}$ hours; however, the group using PP had a lower pain level at the $18^{\text {th }}$ hour. The consumption of opioids in the patients using the PP was reported to be less than that in the patients that did not use the PP at the end of postoperative day 1. The requirement for anti-emetic drugs and blood transfusion by the patients using the PP was lower. Some data obtained during this study support our study. What was different in our study was that the group using PP had lower pain scores in the early postoperative period, and the pain scores at the $24^{\text {th }}$ and $48^{\text {th }}$ hours were similar in both groups. This may be explained by effective relief of pain by us through a multimodal analgesia protocol after the $12^{\text {th }}$ hour in both of the groups. In addition, the use of opioids at the end of $24^{\text {th }}$ and $48^{\text {th }}$ hours was less in the PP + PCA group, which was similar to the other study. Different from that study, we also compared the patients' time to walk and discharge time. The time to walk and discharge time in the group PA + PCA were earlier. The mean of discharge times was higher as compared to other studies, which might be due to sociodemographic characteristics of patients as a major reason. Many patients were from a rural area; therefore, they waited for a complete healing before they departed, and those patients that would use a corset waited for one. Our study had several limitations; for example, we could not compare the complications such as nausea, vomitting, and fever, which are common in the postoperative period, as this was a retrospective study. We also consider that preoperative pain threshold and postoperative pain scores of patients would be effective in assessment; however, we did not carry out such an assessment.

Table 3. Mobilization and discharge time

\begin{tabular}{llll}
\hline & Group pain pump $(\mathrm{n}=49)$ & Group control $(\mathrm{n}=34)$ & $\mathrm{p}$ \\
\hline Walking time (day) & $2.67 \pm 0.99$ & $3.68 \pm 0.94$ & $<0.0001^{\mathrm{a}}$ \\
\hline Discharge time (day) & $8.00 \pm 2.03$ & $10.00 \pm 4.56$ & $\mathbf{0 . 0 4 5}^{\mathrm{a}}$
\end{tabular}

Table 4. Opioid consumption

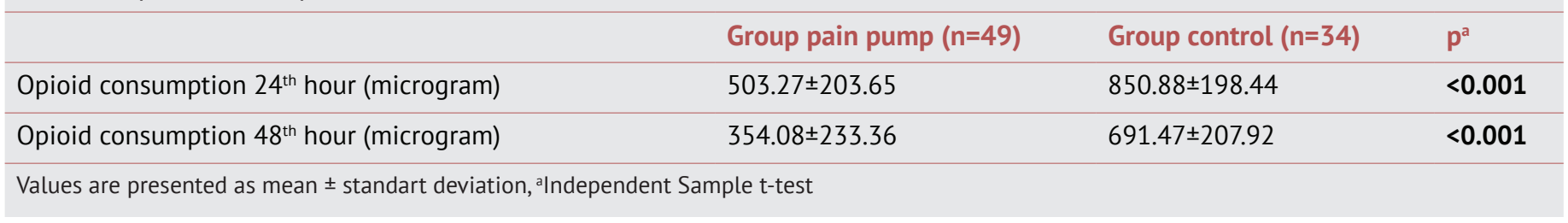




\section{CONCLUSION}

As a result, we observed that the use of PCA in combination with a continuous release PP that was inserted into the incision site helped early postoperative pain control in the patients with AIS and enabled patients to walk earlier and to be discharged earlier. We also demonstrated that the PP reduced postoperative consumption of opioids. We believe that this study would help patients with AIS in pain control through further prospective randomized studies including more patients.

\section{Ethics}

Ethics Committee Approval: The study protocol was approved by the Ethics Committee of Atatürk University (approval number: 27.12.2018.08/11).

Informed Consent: Retrospective study.

Peer-review: Internally peer-reviewed.

\section{Authorship Contributions}

Surgical and Medical Practices: S.Y., Concept: S.Y., Design: S.Y., Data Collection or Processing: S.Y., Analysis or Interpretation: A.A., Literature Search: A.A., Writing: S.Y., A.A.

Conflict of Interest: No conflict of interest was declared by the authors.

Financial Disclosure: The authors declared that this study received no financial support.

\section{REFERENCES}

1. Arms DM, Smith JT, Osteyee J, Gartrell AJO. Postoperative epidural analgesia for pediatric spine surgery. Orthopedics 1998;21:539-44.

2. Blumenthal S, Borgeat $A$, Nadig M, Min K. Postoperative analgesia after anterior correction of thoracic scoliosis: a prospective randomized study comparing continuous double epidural catheter technique with intravenous morphine. Spine 2006;31:1646-51.

3. Elder JB, Hoh DJ, Wang MY. Postoperative continuous paravertebral anesthetic infusion for pain control in lumbar spinal fusion surgery. Spine 2008;33:210-8.
4. Gauger VT, Voepel-Lewis TD, Burke CN, Kostrzewa AJ, Caird MS, Wagner DS, et al. Epidural analgesia compared with intravenous analgesia after pediatric posterior spinal fusion. J Pediatr Orthop 2009;29:588-93.

5. Gulur P, Nishimori M, Ballantyne JC. Regional anaesthesia versus general anaesthesia, morbidity and mortality. Best Pract Res Clin Anaesthesiol 2006;20:249-63.

6. Hong RA, Gibbons KM, Li GY, Holman A, Voepel-Lewis T. A retrospective comparison of intrathecal morphine and epidural hydromorphone for analgesia following posterior spinal fusion in adolescents with idiopathic scoliosis. Paediatr Anaesth 2017;27:91-97.

7. Jones MD, Aronsson DD, Harkins JM, Smail DF, Haugh LD. Epidural analgesia for postoperative pain control in children. J Pediatr Orthop 1998; 18:492-6.

8. Klatt JW, Mickelson J, Hung M, Durcan S, Miller C, Smith JT. A randomized prospective evaluation of 3 techniques of postoperative pain management after posterior spinal instrumentation and fusion. Spine 2013;38:1626-31.

9. Milbrandt TA, Singhal M, Minter C, McClung A, Talwalkar VR, Iwinski $\mathrm{HJ}$, et al. A comparison of three methods of pain control for posterior spinal fusions in adolescent idiopathic scoliosis. 2009;34:1499-503.

10. Milbrandt TA, Singhal M, Minter C, McClung A, Talwalkar VR, Iwinski $\mathrm{HJ}$, et al. A comparison of three methods of pain control for posterior spinal fusions in adolescent idiopathic scoliosis. Spine (Phila Pa 1976) 2009;34:1499-503.

11. Ross PA, Smith BM, Tolo VT, Khemani RGJS. Continuous infusion of bupivacaine reduces postoperative morphine use in adolescent idiopathic scoliosis after posterior spine fusion. Spine (Phila Pa 1976) 2011;36:1478-83.

12. Saudan S, Habre W, Ceroni D, Meyer PA, Greenberg RS, Kaelin A, et al. Safety and efficacy of patient controlled epidural analgesia following pediatric spinal surgery. Paediatr Anaesth 2008;18:132-9.

13. Singh K, Phillips FM, Kuo E, Campbell M. A prospective, randomized, double-blind study of the efficacy of postoperative continuous local anesthetic infusion at the iliac crest bone graft site after posterior spinal arthrodesis: a minimum of 4-year follow-up. Spine (Phila Pa 1976) 2007;32:2790-6.

14. Tirotta CF, Munro HM, Salvaggio J, Madril D, Felix DE, Rusinowski $\mathrm{L}$, et al. Continuous incisional infusion of local anesthetic in pediatric patients following open heart surgery. Paediatr Anaesth 2009; 19:571-6.

15. Turner A, Lee J, Mitchell R, Berman J, Edge G, Fennelly M. The efficacy of surgically placed epidural catheters for analgesia after posterior spinal surgery. Anaesthesia 2000;55:370-3. 